Leilane Camila Ferreira de

Lima Francisco

(ㄱ) https://orcid.org/0000-0003-4945-8939

Alice Correia Barros ${ }^{1}$

Ohttps://orid.org/0000-0002-2627-7185

Mariana da Silva Pacheco²

Ohttps://orcid.org/0000-0002-7599-8157

Antonio Egidio Nardi ${ }^{3}$

(1) https://orcid.org/0000-0002-2152-4669

Verônica de Medeiros Alves'

(D)https://orcid.org/0000-0002-4343-2941

\section{Ansiedade em minorias sexuais e de gênero: uma revisão integrativa}

\author{
Anxiety in sexual and gender minorities: an integrative review
}

DOI: 10.1590/0047-2085000000255

\section{RESUMO}

Objetivo: Evidenciar os fatores predisponentes relativos à ansiedade em minorias sexuais e de gênero na literatura. Métodos: Trata-se de uma revisão integrativa. A busca dos artigos foi realizada em três bases de dados eletrônicas: PubMed/Medline, Scopus e ISI Web of Knowledge. Foram utilizados os descritores "anxiety", "LGBT people", "gay", "bisexual", "lesbian" e "transgender", com textos completos, publicados no período de 2013 a 2018, no idioma inglês, e foi usado o operador boleano AND. Resultados: Foram encontrados 712 artigos. Cinquenta e oito (58) artigos foram selecionados para serem lidos na íntegra e 13 atenderam aos critérios de inclusão desta revisão. As evidências mostram que a população LGBT apresenta maior risco para transtornos mentais, entre eles a ansiedade, quando comparada aos heterossexuais. O aparecimento dos sinais e sintomas de ansiedade estão relacionados com a vergonha e o comportamento evitativo dessa população devido à forte discriminação e à ausência de apoio social e familiar, o que ocasiona altos níveis de angústia. Apenas dois artigos estudaram menores de 18 anos. Conclusões: Os profissionais da saúde devem estar abertos, acolhedores e atentos à saúde mental desse público, visando contribuir com a promoção da saúde, apoio social, familiar e a redução da discriminação.

\section{PALAVRAS-CHAVE}

Ansiedade, minorias sexuais e de gênero, pessoas LGBT.

\section{ABSTRACT}

Objective: To evidence the predisposing factors related to anxiety in sexual and gender minorities in the literature. Methods: This is an integrative review. The search of the articles was conducted in three databases: PubMed/Medline, Scopus and ISI Web of Knowledge. The descriptors "anxiety", "LGBT people", "gay", "bisexual", "lesbian" and "transgender" were used, with complete texts, published between 2013 and 2018, in the English language, boolean operator AND was used. Results: A total of 712 articles were found. Fifty-eight (58) articles were selected to be read in their entirety and 13 met the inclusion criteria of this review. Evidence shows that LGBT people is at higher risk for mental disorders, including anxiety, when compared to heterosexual. The appearance of signs and symptoms of anxiety are related to shame and the avoidant behavior of this population due to the strong discrimination and lack of social and family life, which causes high levels of distress. Only two articles studied minors 18 years old. Conclusions: Health professionals should be welcoming and attentive to health mental health of this public, with a view to contributing to health promotion, social and family support and reduction of discrimination.

\section{KEYWORDS}

Anxiety, sexual and gender minorities, LGBT people. 


\section{INTRODUÇÃO}

No que diz respeito às questões de gênero, pouco se conhece sobre sua origem e história. O que se sabe é que, em torno do ano de 1950, o termo foi introduzido pela primeira vez na ciência por um psicólogo chamado John Money, o qual identificou que nem sempre vai haver relação entre a anatomia sexual de uma pessoa e sua identidade de gênero, ou seja, existem homens e mulheres que compõem a humanidade, mas a forma de ser homem e de ser mulher é resultado de um contexto cultural ${ }^{1,2}$. Nesse sentido, há diferenças entre os conceitos de gênero, identidade de gênero e orientação sexual, descritos a seguir.

O gênero se relaciona ao sexo biológico, nomeando-se de gênero feminino e gênero masculino. Adiciona-se ainda ao termo "gênero" o sentido de que esse é o que diferencia socialmente o ser humano, construído de acordo com suas relações e sofrendo influência de aspectos histórico-culturais³

O conceito de identidade de gênero diz respeito a como um indivíduo se percebe e se identifica, não necessariamente sendo condizente com o seu sexo ou orientação sexual'. Os tipos de identidade de gênero são: cisgênero (quando a pessoa se identifica com o gênero igual ao sexo em que nasceu), transgênero (quando o indivíduo se identifica com o gênero diferente do sexo em que nasceu) e não binário (quando o indivíduo não se identifica e não se sente pertencente a nenhum gênero) 4 .

Compreende-se por orientação sexual a capacidade de cada indivíduo ter atração por outro de gênero diferente, igual ou ambos 5 . Quanto à orientação sexual, um indivíduo pode ser homossexual quando sente atração pelo mesmo sexo, heterossexual quando se atrai pelo sexo oposto, bissexual quando sente atração por ambos os sexos, assexual quando não sente atração por nenhum sexo e pansexual quando se sente atraído por pessoas, independentemente de sua identidade de gênero ou sexo biológico ${ }^{4}$.

Nesse sentido, aqueles que não condizem com os padrões heteronormativos impostos pelo ser humano são vítimas de fobias, o que os torna bastante vulneráveis e repercute em intenso sofrimento, indignação e humilhação ${ }^{6}$. A ausência de respeito, gentileza e de acolhimento no atendimento a esses indivíduos se faz bem presente, o que fragiliza a prática do cuidar?

Pesquisa realizada no Ceará identificou que as principais queixas de saúde dessa população foram tristeza, baixa autoestima e ansiedade, seguidas de depressão e insônia Estudo realizado na Inglaterra, com o objetivo de conhecer a prevalência de problemas de saúde mental na população LGBT, encontrou grande relação da homossexualidade com infelicidade geral, transtorno de ansiedade generalizada, desordens neuróticas, episódios depressivos, transtorno fóbico, além de pensamentos e atos suicidas ${ }^{9}$. Outro estudo com homossexuais na maturidade e na velhice verificou que, entre os transtornos mais presentes, o transtorno de ansiedade generalizada e o transtorno depressivo maior se sobressaíam ${ }^{10}$. O transtorno de ansiedade generalizada é caracterizado por uma preocupação intensa e persistente, ocasionando tensão, inquietação e dificuldade de concentrar-se ${ }^{11}$.

É perceptível que pertencer a esse grupo traz uma intensa sobrecarga psíquica a esses indivíduos, o que os torna propensos ao adoecimento mental. Este, por sua vez, repercute diretamente no prejuízo do desempenho das atividades diárias e no sofrimento do indivíduo ${ }^{4}$.

Dados como esses demonstram a necessidade do conhecimento de fatores predisponentes relativos à ansiedade entre as minorias sexuais e de gênero, para que se possam elaborar estratégias de promoção à saúde e de prevenção do adoecimento mental dessa população. Nesse sentido, este estudo busca responder à questão norteadora: Quais os aspectos predisponentes relativos à ansiedade em minorias sexuais e de gênero? O objetivo é evidenciar os fatores predisponentes relativos à ansiedade em minorias sexuais e de gênero na literatura científica.

\section{MÉTODOS}

Trata-se de uma revisão integrativa. A busca dos artigos foi realizada em três bases de dados eletrônicas: National Library of Medicine, EUA (PubMed/Medline), Scopus (Elsevier) e ISI Web of Knowledge (ISI). Para o levantamento dos estudos, foram utilizados os descritores "anxiety", "LGBT people", "gay", "bisexual", "lesbian" e "transgender", no idioma inglês. Realizou-se a busca pelos descritores cruzados com o operador booleano AND. A busca foi realizada no mês de julho de 2018.

Foram seguidos os seis passos para a elaboração de uma revisão integrativa, sendo eles: elaboração da pergunta de pesquisa, estabelecimento de critérios de inclusão e exclusão dos estudos, categorização dos estudos, avaliação dos estudos incluídos, interpretação dos resultados e apresentação da revisão. No caso da presente pesquisa, a síntese dos resultados permite a incorporação de evidências, melhorando, dessa forma, a assistência à saúde do público em questão 12,13.

Para selecionar a amostra, foram utilizados os seguintes critérios de inclusão: artigos que respondessem à questão norteadora, estudos quantitativos indexados nas bases de dados selecionadas, com textos completos, publicados no período de 2013 a 2018, por serem os artigos mais atuais, no idioma inglês. Foram excluídos artigos de revisão e metanálise.

Para a seleção dos artigos, primeiro foram lidos todos os títulos e selecionados aqueles que tinham relação com a temática; na sequência, foram analisados os resumos dos artigos selecionados na primeira etapa, sendo escolhidos os artigos relacionados com a questão norteadora do estudo, 
para leitura na íntegra; por fim, realizou-se a seleção dos estudos primários, de acordo com os critérios de inclusão previamente definidos.

Para a análise dos dados, foi utilizado um quadro sinóptico (Quadro 1) contendo variáveis que responderam à questão norteadora desta revisão integrativa. Os tópicos de interesse foram: autor, ano, método, amostra, local do estudo, instrumentos utilizados na referida pesquisa e resultados de aspectos associados à ansiedade em minorias sexuais e de gênero.

Quadro 1. Artigos selecionados que mostraram aspectos relativos à ansiedade em minorias sexuais e de gênero

\begin{tabular}{|c|c|c|c|c|}
\hline Autor, ano & Método & Amostra & Local & Instrumentos \\
\hline $\begin{array}{l}\text { Budge } \\
\text { et al., } 2013\end{array}$ & $\begin{array}{l}\text { Quantitativo, } \\
\text { transversal }\end{array}$ & $\begin{array}{l}351 \text { indivíduos transgêneros } \\
\text { (226 mulheres trans e } 125 \\
\text { homens trans) } \\
\text { Média de idade de 40,28 } \\
\text { anos e desvio-padrão } \pm \\
13,85\end{array}$ & $\begin{array}{l}\text { Estados Unidos da } \\
\text { América }\end{array}$ & $\begin{array}{l}\text { Questionário demográfico, Escala } \\
\text { multidimensional de suporte social } \\
\text { percebido (MSPSS), Questionário de } \\
\text { formas de enfrentamento, Escala de } \\
\text { percepção de perda para } \\
\text { transgêneros, Escala de depressão } \\
\text { do Centro de Estudos } \\
\text { Epidemiológicos (CES-D), Inventário } \\
\text { de Ansiedade (BAl) }\end{array}$ \\
\hline $\begin{array}{l}\text { Choi et al., } \\
2013\end{array}$ & $\begin{array}{l}\text { Quantitativo, } \\
\text { transversal }\end{array}$ & $\begin{array}{l}1.196 \text { participantes } \\
\text { Aproximadamente } 403 \\
\text { afro-americanos, } 393 \\
\text { asiáticos e do Pacífico e } 400 \\
\text { latinos } \\
\text { Idades entre } 18 \text { e } 83 \text { anos }\end{array}$ & $\begin{array}{l}\text { Estados Unidos da } \\
\text { América }\end{array}$ & $\begin{array}{l}\text { Questionário de características } \\
\text { demográficas, Questionários de } \\
\text { experiências de racismo e } \\
\text { homofobia, Escala de depressão do } \\
\text { Centro de Estudos Epidemiológicos, } \\
\text { Subescala de Ansiedade de } 6 \text { itens } \\
\text { do Brief Symptom Inventory }\end{array}$ \\
\hline
\end{tabular}

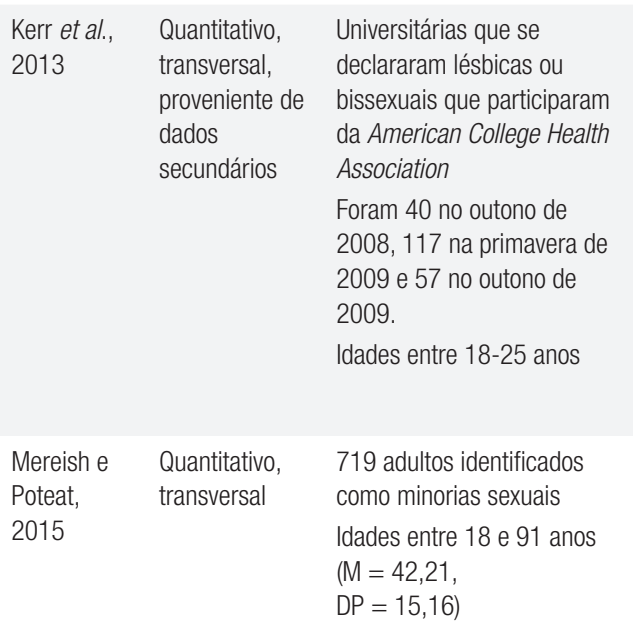

Wadsworth

Q Quantitativo,

Hayes-

Skelton,

2015

\section{Estados Unidos da Questionário ACHA-NCHA II (National América College Health Assessment II)}

Estados Unidos da América

$\mathrm{DP}=15,16$

\section{Resultados}

$40,4 \%$ (n 91) mulheres transexuais relataram sintomatologia de ansiedade significativa, enquanto $47,5 \%$ (n 64) de homens transgêneros relataram sintomatologia de ansiedade significativa.

Experiências passadas de racismo e homofobia advindos da sociedade e de amigos heterossexuais foram associadas com a ansiedade. 0 nível de ansiedade foi maior entre os latinos, seguidos dos do Pacífico e afro-americanos.

A associação do racismo na comunidade gay com ansiedade diferiu por raça/etnia e foi estatisticamente significante apenas para os do Pacífico.

A homofobia percebida dentro da família não foi associada com a ansiedade.

As mulheres bissexuais relataram o pior estado de saúde mental em todas as áreas estudadas, incluindo ansiedade, raiva, sintomas depressivos, autolesão, e ideação suicida e tentativas. Ambas as mulheres bissexuais e lésbicas tiveram uma probabilidade maior de ter problemas de saúde mental quando comparados com mulheres heterossexuais. Lésbicas e mulheres bissexuais utilizaram significativamente mais serviços de saúde mental do que as mulheres heterossexuais.

\section{Vergonha foi associada a relacionamentos mais pobres com outras pessoas e teve fortes associações diretas com angústia psicológica (ansiedade e depressão). \\ Relacionamentos mais pobres associaram- se a níveis mais altos de solidão e a solidão estava associada a sofrimento psicológico (ansiedade e depressão). \\ Escala de Assédio, Rejeição e Revisada de Homofobia Internalizada, Subescala de motivação por ocultação de identidade LGBT, Subescala da vergonha do questionário Personal Feelings-2, Índice de Saúde} Relacional, Escala de Solidão De Jong Gierveld, Subescalas de depressão e ansiedade da Escala-21 de Depressão, Ansiedade e Estresse, Inventário de Sintomas Físicos de Cohen-Hoberman

Estados Unidos da Escala de Ansiedade Social de América Liebowitz (LSAS-SR)
Os individuos que se identificaram como bissexuais classificaram níveis significativamente mais elevados de ansiedade do que heterossexuais e lésbicas/gays. Isso tem relação com a exclusão, a falta de suporte social e 0 estigma. 


\begin{tabular}{|c|c|c|c|c|}
\hline Autor, ano & Método & Amostra & Local & Instrumentos \\
\hline $\begin{array}{l}\text { Yang et al., } \\
2016\end{array}$ & $\begin{array}{l}\text { Quantitativo, } \\
\text { transversal }\end{array}$ & $\begin{array}{l}209 \text { mulheres transgêneros } \\
\text { Idades entre } 18 \text { e } 45 \text { anos } \\
(\mathrm{M}=26,64, \mathrm{DP}=4,32)\end{array}$ & China & $\begin{array}{l}\text { Escala de Ansiedade de Zung (SAS), } \\
\text { Características sociodemográficas, } \\
\text { Questões sobre parceiro sexual, } \\
\text { discriminação, divulgação da } \\
\text { identidade de gênero e } \\
\text { conhecimento sobre a prevenção do } \\
\text { HIV e dos serviços de saúde, Escala } \\
\text { multidimensional de apoio social } \\
\text { percebido }\end{array}$ \\
\hline
\end{tabular}

Resultados

A prevalência de sintomas de ansiedade em mulheres transgêneros chinesas foi de $34,5 \%$, mostrando um nível consideravelmente elevado de sintomas da ansiedade. As análises de regressão indicaram que a ansiedade estava associada a parcerias casuais, discriminação de amigos e apoio social.

Verificou-se também que foram expostos a importantes desafios de transição, como a parceria sexual de alto risco, a discriminação excessiva e a redução do apoio social. Os sintomas de ansiedade foram mais bem previstos pela ausência ou presença de um parceiro casual, discriminação de amigos e apoio social, em vez da divulgação de sua identidade de gênero, conhecimento da prevenção do HIV e serviço de saúde.

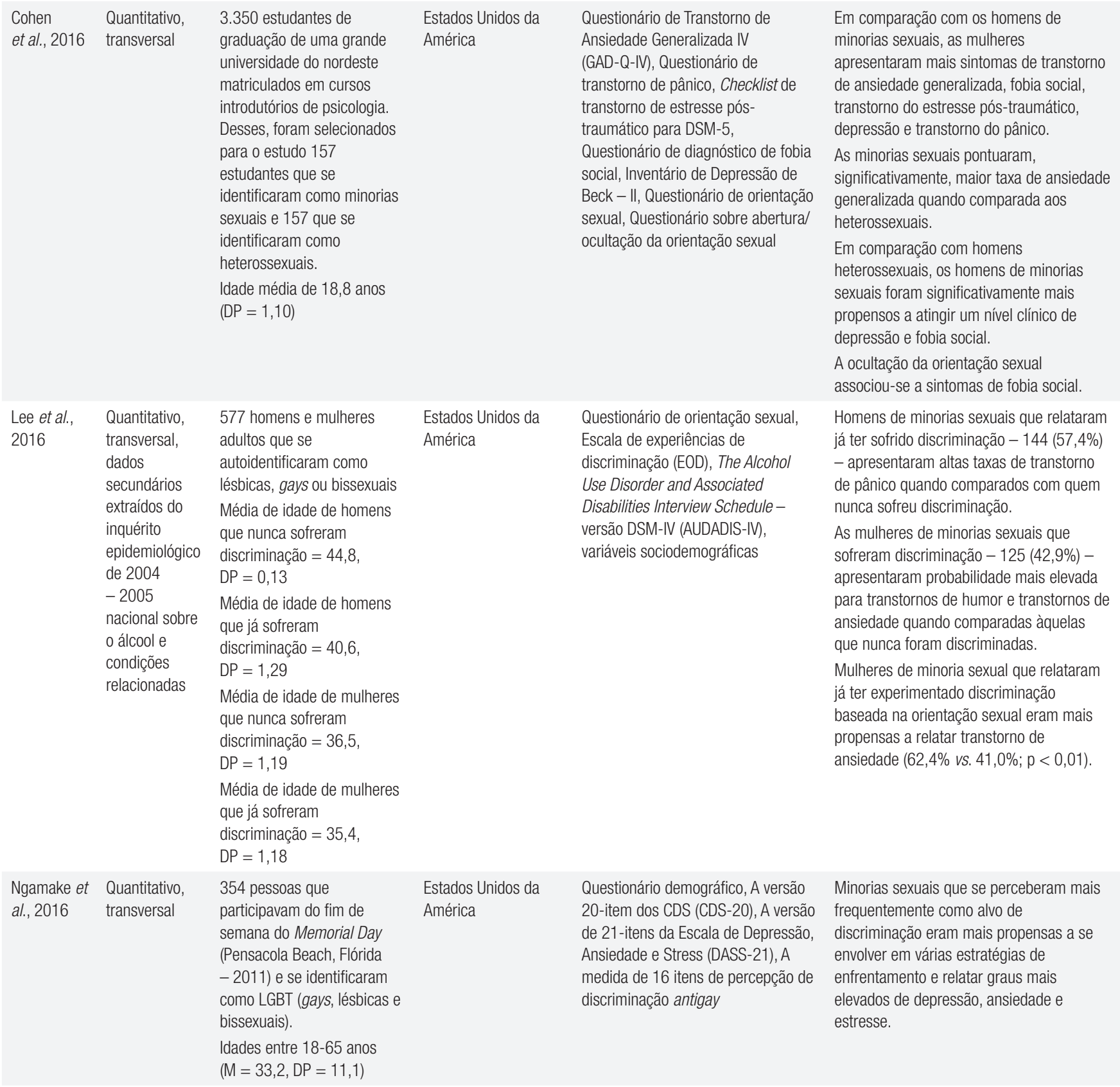




\begin{tabular}{|c|c|c|c|c|c|}
\hline Autor, ano & Método & Amostra & Local & Instrumentos & Resultados \\
\hline $\begin{array}{l}\text { Reisner et } \\
\text { al., } 2016\end{array}$ & $\begin{array}{l}\text { Quantitativo, } \\
\text { coorte, } \\
\text { longitudinal }\end{array}$ & $\begin{array}{l}7.831 \text { jovens de minorias } \\
\text { sexuais e de gênero e } \\
\text { heterossexuais }\end{array}$ & $\begin{array}{l}\text { Estados Unidos da } \\
\text { América }\end{array}$ & $\begin{array}{l}\text { Medida de identidade de gênero } \\
\text { Escala de } 10 \text { itens do Centro de } \\
\text { Estudos Epidemiológicos de }\end{array}$ & $\begin{array}{l}\text { Ao se compararem as minorias de gênero } \\
\text { com as não minorias de gênero, o primeiro } \\
\text { grupo apresentou maior nível de ansiedade. }\end{array}$ \\
\hline
\end{tabular}

\begin{tabular}{|c|c|c|c|c|c|}
\hline $\begin{array}{l}\text { Shearer et } \\
\text { al., } 2016\end{array}$ & $\begin{array}{l}\text { Quantitativo, } \\
\text { transversal }\end{array}$ & $\begin{array}{l}2.513 \text { jovens que foram } \\
\text { convidados a participar de } \\
\text { um projeto de prevenção ao } \\
\text { suicídio por meio de } 10 \\
\text { unidades de atenção } \\
\text { primária } \\
\text { Idades entre } 14 \text { e } 24 \text { anos } \\
(\mathrm{M}=17,24 \text {; DP }=2,86)\end{array}$ & $\begin{array}{l}\text { Estados Unidos da } \\
\text { América }\end{array}$ & $\begin{array}{l}\text { Escalas de Tela de Saúde } \\
\text { Comportamentais (Behavioral Health } \\
\text { Screen scales - BHS) }\end{array}$ & $\begin{array}{l}\text { As mulheres bissexuais e queer } \\
\text { apresentaram maior nível de depressão, } \\
\text { ansiedade e estresse do que mulheres } \\
\text { heterossexuais. } \\
\text { Homens gays e bissexuais apresentaram } \\
\text { maior nível de depressão e estresse. Os } \\
\text { homens gays também exibiram maiores } \\
\text { escores na subescala de ansiedade do que } \\
\text { os homens heterossexuais. }\end{array}$ \\
\hline $\begin{array}{l}\text { Bränström, } \\
2017\end{array}$ & $\begin{array}{l}\text { Quantitativo, } \\
\text { longitudinal, } \\
\text { coorte }\end{array}$ & $\begin{array}{l}30.730 \text { indivíduos de } 18 \\
\text { anos de idade ou mais } \\
\text { Idades de gays/lésbicas } \\
(\mathrm{M}=45,8, \mathrm{DP}=15,8) \\
\text { Idades de bissexuais } \\
(\mathrm{M}=38,9, \mathrm{DP}=17,8) \\
\text { Idades de heterossexuais } \\
(\mathrm{M}=50,9, \mathrm{DP}=18,0)\end{array}$ & Suécia & $\begin{array}{l}\text { Questionário semiestruturado - } \\
\text { Questões sobre identidade de } \\
\text { gênero e orientação sexual, } \\
\text { presença de companheiro, } \\
\text { vitimização e agressão, apoio social, } \\
\text { educação e renda, tratamento em } \\
\text { saúde mental }\end{array}$ & $\begin{array}{l}\text { Gays, lésbicas e bissexuais eram mais } \\
\text { propensos a relatar experiências de } \\
\text { vitimização e ameaça de agressões que os } \\
\text { heterossexuais. } \\
\text { Heterossexuais relataram maior apoio } \\
\text { social que os outros grupos de minorias } \\
\text { sexuais. } \\
\text { Durante o período de acompanhamento de } \\
12 \text { meses, } 5,8 \% \text { dos gays/lésbicas, } 13,3 \% \\
\text { dos bissexuais e } 3,2 \% \text { dos heterossexuais } \\
\text { tinham procurado serviços de saúde mental } \\
\text { pelo menos uma vez. } \\
\text { Gays/lésbicas e bissexuais tinham um risco } \\
\text { elevado de serem tratados para ansiedade } \\
\text { em comparação com os heterossexuais. }\end{array}$ \\
\hline $\begin{array}{l}\text { Wang et al., } \\
2018\end{array}$ & $\begin{array}{l}\text { Quantitativo, } \\
\text { transversal }\end{array}$ & $\begin{array}{l}\text { Os participantes foram } \\
\text { recrutados por meio de } \\
\text { propagandas na internet, } \\
\text { incluindo Facebook, sistemas } \\
\text { de quadro de avisos e as } \\
\text { homepages de cinco } \\
\text { promoção de saúde e } \\
\text { centros de aconselhamento } \\
\text { para a comunidade LGBT de } \\
\text { agosto de } 2015 \text { a julho de } \\
2017 . \text { Um total de } 500 \text { gays } \\
\text { ( } \mathrm{n}=371 \text { ) ou bissexuais } \\
\text { ( } \mathrm{n}=129 \text { ) homens de } \\
\text { Taiwan. } \\
\text { Idades entre } 20 \text { e } 25 \text { anos }\end{array}$ & Taiwan & $\begin{array}{l}\text { Versão chinesa do questionário de } \\
\text { experiência de bullying escolar, } \\
\text { Questionário de experiências de } \\
\text { cyberbullying, versão em chinês } \\
\text { mandarim do Centro de Estudos } \\
\text { Epidemiológicos-Escala de } \\
\text { Depressão (MC-CES-D), Subescala } \\
\text { do Inventário de Ansiedade } \\
\text { Traço-Estado (IDATE). Subescala } \\
\text { de dor física do questionário de } \\
\text { qualidade de vida de adolescentes } \\
\text { taiwaneses, Dados } \\
\text { sociodemográficos }\end{array}$ & $\begin{array}{l}\text { Vítimas de qualquer tipo de bullying } \\
\text { homofóbico na infância tiveram depressão, } \\
\text { ansiedade e dor física mais severas na } \\
\text { idade adulta emergente do que as não } \\
\text { vítimas. As vítimas do bullying homofóbico } \\
\text { tradicional e cibernético tiveram uma } \\
\text { ansiedade mais severa na idade adulta do } \\
\text { que as vítimas de apenas bullying } \\
\text { homofóbico tradicional ou cibernético. } \\
0 \text { apoio da família moderou os níveis atuais } \\
\text { de ansiedade e dor física na idade adulta } \\
\text { emergente entre homens gays e bissexuais. }\end{array}$ \\
\hline
\end{tabular}

\section{RESULTADOS}

A figura 1 ilustra o processo de seleção dos artigos. Foi encontrado um total de 712 artigos, sendo 19 na base de dados ISI Web of Knowledge, 279 na PubMed/Medline e 414 na Scopus. Após descartar os 217 estudos duplicados, 495 artigos foram, inicialmente, analisados pelos seus respectivos títulos e resumos, sendo excluídos 437. Cinquenta e oito (58) artigos foram selecionados para serem lidos na íntegra. Após esse processo, treze (13) artigos atenderam aos critérios de inclusão que avaliaram os aspectos predisponentes relativos à ansiedade em minorias sexuais e de gênero (Figura 1).
Depressão

Subescala de 9 itens de preocupação/Escala de Ansiedade (RCMAS)

Características demográficas

Ecalas de Tela de Saúde Comportamentais (Behavioral Health Screen scales - BHS) apresentaram maior nível de depressão, ansiedade e estresse do que mulheres rossexuais.

maior nível de depressão e estresse. Os homens gays também exibiram maiores escores na subescala de ansiedade do que ens heterossexuais. vitimização e ameaça de agressões que os heterossexuais. sexuais.

12 meses, $5,8 \%$ dos bissexuais e $3,2 \%$ dos heterossexuais pelo menos uma vez.

Gays/lésbicas e bissexuais tinham um risco elevado de serem tratados para ansiedade homofóbico na infância tiveram depressão, ansiedade e dor física mais severas na ade adulta emergente do que as não ansiedade mais severa na idade adulta do que as vítimas de apenas bullying homofóbico tradicional ou cibernético.

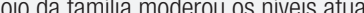
de ansiedade e dor física na idade adulta taiwaneses, Dados sociodemográficos
Os estudos foram realizados nos seguintes países: Estados Unidos ${ }^{14-23}$, China ${ }^{24}$, Taiwan ${ }^{25}$ e Suécia ${ }^{26}$. Foram realizados três estudos em 2013, três em 2015, cinco em 2016, um em 2017 e um em 2018 (Quadro 1).

A amostra dos estudos selecionados era composta pelas diversas orientações sexuais e identidades de gênero, a saber gays, lésbicas, bissexuais, transgêneros, queer ou heterossexuais, sendo feitas comparações entre os dois grupos no que diz respeito aos níveis de ansiedade e seus aspectos relativos. As minorias sexuais foram avaliadas quanto à presença de ansiedade. A menor amostra foi composta por 180 lésbicas, gays, bissexuais e heterossexuais ${ }^{17}$, e a maior, 


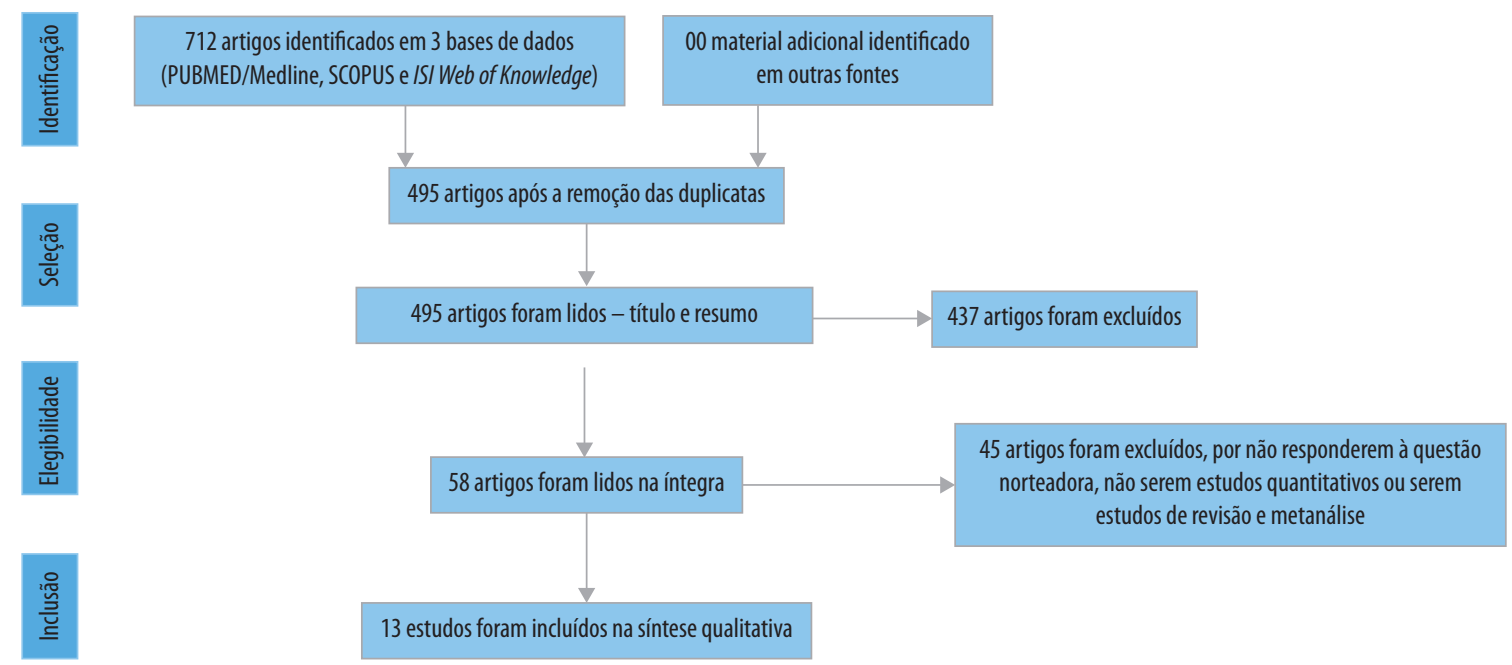

Figura 1. Seleção dos artigos por descritores nas bases de dados.

por 30.730 indivíduos pertencentes a minorias sexuais e de gênero, sendo comparados a indivíduos heterossexuai ${ }^{26}$.

Os estudos selecionados apresentaram diferentes métodos e todos conseguiram medir o que estava proposto. Dentre os métodos utilizados nos estudos selecionados, destacaram-se os estudos transversais e quantitativos, os quais incluíam as minorias sexuais e de gênero na população a ser estudada, não comparando o desfecho ansiedade com os grupos heterossexuais $14,18-20,22,24-25$.

Quanto à idade dos participantes dos estudos selecionados, apenas dois estudos ${ }^{21,23}$ avaliaram sinais e sintomas de ansiedade em minorias sexuais e de gênero menores de 18 anos, o que reforça a necessidade de se realizarem pesquisas com o público adolescente.

Os artigos selecionados apresentaram diversos instrumentos para avaliar a relação da ansiedade nas minorias sexuais e de gênero, sendo eles: Escala de Beck - Inventário de Ansiedade (BAI) ${ }^{14}$; Questionário de Transtorno de Ansiedade Generalizada IV (GAD-Q-IV) ${ }^{15}$; Questionário ACHA-NCHA ॥ (National College Health Assessment II) ${ }^{16}$; Escala de Ansiedade Social de Liebowitz (LSAS-SR) ${ }^{17}$; The Alcohol Use Disorder and Associated Disabilities Interview Schedule - Versão DSM-IV (AUDADIS-IV) ${ }^{18}$; Escala de Depressão, Ansiedade e Stress (DASS21)19; As subescalas de depressão e ansiedade da Escala-21 de Depressão, Ansiedade e Estresse ${ }^{20}$; Subescala de 9 itens de Preocupação/Escala de Ansiedade (RCMAS)21; Subescala de Ansiedade de 6 itens - Brief Symptom Inventory (BSI)22; Escala de Saúde Comportamental (Behavioral Health Screen scales BHS ${ }^{23}$; Escala de Ansiedade de Zung (SAS) 24 ; Inventário de Ansiedade Traço-Estado (IDATE) ${ }^{25}$; questionário semiestruturado - questões sobre tratamento em saúde mental26 (Tabela 1).

Os artigos selecionados permitiram evidenciar que:

- A população LGBT apresentou sintomas em níveis mais elevados de transtornos ansiosos, entre eles o transtorno de ansiedade generalizada, fobia social, transtono do estresse pós-traumático e pânico, seguidos de depressão, quando comparada aos heterossexuais ${ }^{15}$, sendo o risco de desenvolver ansiedade duas a três vezes maior que nos heterossexuais ${ }^{21}$;

- Indivíduos transgêneros apresentaram alta taxa de ansiedade ${ }^{14,24}$. Gays, queer e bissexuais apresentaram maior nível de ansiedade, sendo esses mais acometidos pelo transtorno ${ }^{23}$. Dois artigos ${ }^{16,17}$ identificaram duas vezes mais chances de bissexuais apresentarem ansiedade do que heterossexuais e, ao comparar grupos bissexuais com lésbicas e gays, os primeiros eram o grupo de maior risco.

Observou-se que, independentemente do seguimento da orientação sexual entre as minorias sexuais e de gênero, o surgimento da ansiedade tem forte relação com a falta de suporte, o estigma da sociedade, família e amigos e a vergonha por não seguir padrões heteronormativos, o que faz com que o indivíduo oculte a orientação sexual 15,17-20,24,25.

Em estudo realizado na Suécia, jovens gays, lésbicas e bissexuais relataram entre duas e três vezes mais falta de apoio social em comparação com heterossexuais ${ }^{26}$. Um estudo destacou que a homofobia somada ao racismo, principalmente se for advinda de amigos e da sociedade de forma geral, está relacionada ao desenvolvimento de ansiedade ${ }^{22}$. Nessa pesquisa, não houve relação significativa entre o estigma proveniente da família e o desenvolvimento de ansiedade ${ }^{22}$.

\section{DISCUSSÃO}

Observou-se que houve um avanço nas políticas públicas voltadas para a população LGBT, porém a efetivação delas ainda se configura como desafiadora. Tudo isso se deve à 
contextualização histórica do preconceito e da discriminação. Por se distanciar daquilo que a sociedade prega, que é atribuir aos homens papéis sexuais e culturais, as minorias sexuais e de gênero tornam-se alvo de violência psicológica, como humilhação, cobrança para mudança de comportamento, rejeição e isolamento do convívio social ${ }^{8}$.

Isso proporciona a vivência da angústia entre esses indivíduos, fazendo com que eles não encontrem o apoio social e adotem comportamentos contrários ao de aprender novas habilidades e de buscar meios alternativos que promovam felicidade e bem-estar ${ }^{14}$. Vê-se a gravidade do problema ao se observar que uma em cada cinco jovens lésbicas não recebiam suporte social no estudo de Bränström ${ }^{26}$.

A vergonha de revelar a orientação sexual promove relações sociais pobres, ocasionando angústia, sofrimento e isolamento por parte das minorias de gênero ${ }^{20}$. Somado a isso, torna-se um desafio para as minorias sexuais e de gênero decidirem se revelarão sua orientação sexual e a forma como isso deverá acontecer, o que causa intenso sofrimento ${ }^{27}$. Tudo isso prejudica sua saúde mental, contribuindo para o aumento dos sinais e sintomas de ansiedade. Dessa forma, assumir para a sociedade a orientação sexual ocasiona a imposição de limites aos seus desejos, deixando esses indivíduos mais propensos ao desrespeito, à discriminação e à relação do ser LGBT à doença, ao desvio, ao crime e à oposição 28 .

A maioria dos estudos sobre a ansiedade em minorias sexuais e de gênero tem sido realizada nos Estados Unidos. Isso reforça a necessidade de pesquisas em outros países e continentes, de forma a melhor identificar o adoecimento mental dessa população e traçar intervenções que visem à redução dessa problemática, de acordo com as características de cada região. Além disso, diante da complexidade do tema e da pequena quantidade de artigos identificados nesta revisão, percebe-se a necessidade de se realizarem mais estudos voltados a essa população.

Vale destacar, ainda, a importância de se realizarem estudos transversais nos quais se comparem os grupos de minorias sexuais e de gênero com os grupos heterossexuais, com o objetivo de evidenciar quem são os mais acometidos pelo desfecho ansiedade. Quanto aos instrumentos utilizados nos estudos selecionados, esses foram fiéis para o rastreio de ansiedade, exceto um questionário semiestruturado utilizado por Bränström² ${ }^{26}$, no qual foram elaborados questionamentos que diziam respeito ao tratamento em saúde mental, não investigando a fundo os sinais e sintomas de ansiedade, o que reforça a necessidade de se utilizarem questionários validados em pesquisas científicas.

Autores relataram, em seu estudo, que o estigma proveniente da família pouco teve efeito sobre a ansiedade, sugerindo que ele pôde ser compensado pelos benefícios oferecidos pela família, por exemplo, o apoio financeiro, ou pelo fato de as minorias sexuais e de gênero já não dependerem financeiramente da família e não terem mais contato com ela, devido à não aceitação de sua orientação sexual ${ }^{22}$. Outros autores revelaram que as variáveis "baixo apoio familiar" vs. "vítimas de homofobia" foram significativamente associadas à ansiedade ${ }^{25}$. Um estudo identificou que a ausência de apoio social, especialmente do familiar, é mais fortemente associada a problemas de saúde mental do que a exposição à violência e à discriminação ${ }^{26}$. Isso indica que o apoio familiar pode moderar os níveis de ansiedade.

Um estudo identificou que indivíduos LGBT que recebiam uma renda maior apresentavam menores níveis de ansiedade ${ }^{19}$. Porém, vê-se que não ser aceito socialmente causa certo desconforto, e isso sinaliza a importância de se dar maior atenção à saúde mental dessa população.

Esse fato, somado à dúvida sobre a reação das pessoas e, principalmente, dos familiares, causa intensa angústia, pois há o receio de envergonhá-los e decepcioná-los e até mesmo da expulsão de seu domicílio29. Essa pessoa, por medo da repressão e rejeição, passa a viver de acordo com as normas impostas, buscando satisfazer apenas os desejos da população, o que causa intenso sofrimento e interfere diretamente no autoconhecimento, na sua saúde e em suas relações pessoais ${ }^{30}$.

Além disso, indivíduos que sofrem discriminação e passam a usar/abusar de drogas para amenizar esse sofrimento tornam-se bem mais propensos a relatar transtornos mentais, entre eles a ansiedade ${ }^{19}$.

Indivíduos transgêneros, por temor da opinião dos demais com quem convivem, acabam por esconder fatos da transição de gênero, fazendo com que isso restrinja seu acesso aos serviços de saúde ${ }^{24}$. Isso prejudica seu bem-estar físico e emocional e reflete a necessidade de sensibilizar a sociedade, os profissionais da saúde, bem como as próprias minorias sexuais.

Particularmente, os jovens bissexuais e queer são um grupo de maior vulnerabilidade para problemas de saúde mental do que heterossexuais ou lésbicas/gays, devido à sobrecarga e aos desafios que esses grupos enfrentam²3. Bissexuais e queer são vítimas de intenso sofrimento devido ao fato de a população em geral acreditar que elesnsão confusos em relação à atração e à orientação sexual ${ }^{16,23}$.

Vale salientar que um estudo identificou que as lésbicas e bissexuais utilizavam mais os serviços de saúde mental do que as mulheres heterossexuais ${ }^{18}$. Porém, outra pesquisa comenta que as minorias sexuais e de gênero ainda estão bastante distantes dos serviços de saúde ${ }^{8}$. Isso se deve ao fato da ausência de respeito, gentileza e acolhimento no atendimento a esses indivíduos, o que fragiliza a prática do cuidar?

Uma pesquisa identificou que os profissionais de saúde não são capacitados para lidar com as questões de saúde da população em questão, o que faz com que sua prática seja pautada pela ausência de sensibilidade e de profissionalismo, refletindo na ausência das minorias sexuais e de gênero nos serviços de saúde e no aumento da vulnerabilidade ao 
adoecimento físico e mental ${ }^{31}$. Dessa forma, a iniciativa de profissionais que respeitem, que saibam lidar com essa demanda e que criem grupos de apoio para essa população pode aproximá-la dos serviços de saúde.

Outro fator que pode interferir na qualidade de vida da população LGBT e na autoafirmação de sua identidade de gênero é a idade. Apenas dois artigos ${ }^{21,23}$ estudaram pessoas menores de 18 anos, sendo essa idade um momento complexo e de transição para a fase adulta e ainda de grande dependência financeira dos pais. Isso pode contribuir com o sofrimento mental dessa população, caso não encontre o apoio necessário da família e da sociedade para lidar com sua própria aceitação.

\section{CONCLUSÕES}

A população LGBT apresenta maior risco para transtornos mentais, entre eles a ansiedade. $O$ aparecimento dos sinais e sintomas de ansiedade estão relacionados com a vergonha e o isolamento dessa população devido à forte discriminação e à ausência de apoio social e familiar, o que ocasiona altos níveis de angústia. O apoio social e familiar e a redução da discriminação podem proteger esses indivíduos contra o desenvolvimento da ansiedade. Isso sinaliza a necessidade de mudança de comportamento social e cultural.

Sendo assim, elaborar estratégias de prevenção ao estigma, orientar familiares quanto aos fatores de risco e à importância do apoio e capacitar estudantes e profissionais de saúde para que possam ter um olhar voltado a essa população, aproximando-a dos serviços de saúde e trabalhando fatores de proteção, pode contribuir com a amenização do adoecimento mental. Além disso, faz-se necessário utilizar as práticas integrativas e complementares como forma de cuidado em saúde mental, de forma a amenizar os sintomas de ansiedade e melhorar a qualidade de vida dessa população.

Sugere-se também que se elaborem pesquisas direcionadas às minorias sexuais e de gênero, principalmente voltadas a estratégias de cuidado que trabalhem na prevenção do adoecimento mental ou que visem à diminuição de sinais e sintomas de ansiedade e demais transtornos.

\section{CONTRIBUIÇÕES INDIVIDUAIS}

\section{Leilane Camila Ferreira de Lima Francisco, Alice Cor- reia Barros e Mariana de Silva Pacheco - Participaram da construção e formação deste estudo.}

\footnotetext{
Antônio Egídio Nardi e Verônica de Medeiros Alves Contribuíram com a análise, interpretação dos dados e revisão crítica do artigo.

Todos os autores revisaram criticamente o seu conteúdo intelectual e aprovaram a versão final a ser publicada.
}

\section{CONFLITO DE INTERESSES}

Os autores declaram não haver conflito de interesses.

\section{REFERÊNCIAS}

1. Araujo LM, Penna LHG. A relação entre sexo, identidades sexual e de gênero no campo da saúde da mulher. Rev Enferm UERJ. 2014;22(1):134-8.

2. Lattanzio FF, Ribeiro PC. Nascimento e primeiros desenvolvimentos do conceito de gênero. Psicol Clin. 2018;30(3):409-25.

3. Reis N, Pinho R. Gêneros não binários: identidades, expressões e educação. Rev Reflex Ação. 2016;24(1):7-25

4. Brasil. Manual orientador sobre diversidade. [Internet]; 2018. Disponível em: https://www. mdh.gov.br/todas-as-noticias/2018/dezembro/ministerio-lanca-manual-orientador-dediversidade/copy_of_ManualLGBTDIGITAL.pdf. Acesso em: 28 nov. 2019.

5. Melo TGR, Sobreira MVS. Identidade de gênero e orientação sexual: perspectivas literárias. Temas em Saúde. 2018;18(3):366-88.

6. Couto Junior DR, Oswald MLMB, Pocahy FA. Gênero, sexualidade e juventude(s). Civitas Rev Ciênc Soc. 2018;18(1):124-37.

7. Bittencourt D, Fonseca D, Segundo M. Acesso da população LGBT moradora de favelas aos serviços públicos de saúde: entraves, silêncios e perspectivas. Conexões Psi. 2014;2(2):60-85.

8. Albuquerque GL, Parente JS, Belém JM, Garcia, CL. Violência psicológica em lésbicas, gays, bissexuais, travestis, e transexuais no interior do Ceará, Brasil. Saúde Debate. 2016;40(109):100-11.

9. Chakraborty A, McManus S, Brugha TS, Bebbington P, King M. Mental health on the nonheterosexual population of England. Br J Psychiatry. 2011;198(2):143-8.

10. Ceará AT, Dalgalarrondo P. Transtornos mentais, qualidade de vida e identidade em homossexuais na maturidade e velhice. Rev Psiquiatr Clin. 2011;37(3):118-23.

11. Araújo AC, Lotufo Neto F. A nova classificação americana para os transtornos mentais - 0 DSM-5. Rev Bras Ter Comport Cogn. 2014;16(1):67-82.

12. Mendes KDS, Silveira RCCP, Galvão CM. Revisão integrativa: método de pesquisa para a incorporação de evidências na saúde e na enfermagem. Texto Contexto Enferm. 2008;17(4):758-64.

13. Whittemore R, Knafl K. The integrative review: updated methodology. J Adv Nurs. 2005;52(5):546-53.

14. Budge SL, Adelson JL, Howard KAS. Anxiety and depression in transgender individuals: the roles of transition status, loss, social support, and coping. J Consult Clin Psychol. 2013;81(3):545-57.

15. Cohen JM, Blasey C, Taylor BC, Weiss BJ, Newman MG. Anxiety and related disorders and concealment in sexual minority young adults. Behav Ther. 2016;47(1):91-101.

16. Kerr DL, Santurri L, Peters PA. Comparison of lesbian, bisexual, and heterosexual college undergraduate women on selected mental health issues. J Am Coll Health. 2013;61(4):185-94.

17. Wadsworth LP, Hayes-Skelton SA. Differences among lesbian, gay, bisexual, heterosexual individuals, and those who reported an other identity on an open-ended response on levels of social anxiety. Psychol Sex Orientat Gend Divers. 2015;2(2):181-7.

18. Lee JH, Gamarel KE, Bryant KJ, Zaller ND, Operario D. Discrimination, mental health, and substance use disorders among sexual minority populations. LGBT Health. 2016;3(4):258-65.

19. Ngamake ST, Walch SE, Raveepatarakul J. Discrimination and sexual minority mental health: mediation and moderation effects of coping. Psychology of Sexual Orientation and Gender Diversity. 2016;3(2):213-26.

20. Mereish EH, Poteat VP. A relational model of sexual minority mental and physical health: the negative effects of shame on relationships, Ioneliness, and health. J Couns Psychol. 2015;62(3):425-37.

21. Reisner SL, Katz-Wise SL, Gordon AR, Corliss HL, Austin SB. Social epidemiology of depression and anxiety by gender identity. J Adolesc Health. 2016;59(2):203-8.

22. Choi K, Paul J, Ayala J, Boylan R, Gregorich SE. Experiences of discrimination and their impact on the mental health among african american, asian and pacific islander, and latino men who have sex with men. Am J Public Health. 2013;103(5):868-74. 
23. Shearer A, Herres J, Kodish T, Squitieri H, James K, Russon J, et al. Differences in mental health symptoms across lesbian, gay, bisexual, and questioning youth in primary care settings. J Adolesc Health. 2016;59(1):38-43.

24. Yang X, Wang L, Song W, Hao C, Zhou J, Zhang Q, et al. A cross-sectional study of associations between casual partner, friend discrimination, social support and anxiety symptoms among Chinese transgender women. J Affect Disord. 2016;203:22-9.

25. Wang C, Chen MH, Chang YP, Yen CF. Effects of traditional and cyber homophobic bullying in childhood on depression, anxiety, and physical pain in emerging adulthood and the moderating effects of social support among gay and bisexual men in Taiwan. Neuropsychiatr Dis Treat. 2018;14:1309-17.

26. Bränström R. Minority stress factors as mediators of sexual orientation disparities in mental health treatment: a longitudinal population-based study. J Epidemiol Community Health. 2017;71(5):446-52.
27. Yarns BC, Abrams JM, Meeks TW, Sewell DD. The mental health of older LGBT adults. Curr Psychiatry Rep. 2016;18(6):1-11.

28. David DEH, Bruns MAT. Mundo-vida travesti: abordagem fenomenológica das travestilidades. Temas Psicol. 2015;23(3):521-33.

29. Gomes AM, Reis AF, Kurashige KD. A violência e o preconceito: as formas da agressão contra a população LGBT em Mato Grosso do Sul. Caderno Espaço Feminino. 2013;26(2):30-43.

30. Zakabi D. Clínica LGBT: contribuições do psicodrama para superação do estigma e da discriminação. Rev Bras Psicodrama. 2014;22(2):6-14.

31. Luvuno ZP, Mchunu G, Ncama B, Ngidi H, Mashamba-Thompson T. Evidence of interventions for improving healthcare access for lesbian, gay, bisexual and transgender people in South Africa: A scoping review. Afr J Prim Health Care Fam Med. 2019;11(1):1-10. 